(Aus dem I. anatomischen Institut der Universitiit Berlin.)

\title{
Ueber einen sehr jungen Anadidymus des Hühnchens.
}

Von

Dr. Erich Hoffimann, Unteraryt.

Hierzu Tatel VIIr.

\section{Einleitung.}

Trotz der zahlreichen Untersuchungen, welche besonders in den letzten Jahrzehnten iiber den Ursprung und das Wesen der Doppelbildungen angestellt worden sind, ist die Frage nach ihrer Entstchung immer noch eine viel umstrittene. Wiahrend sich früher nur zwei Theoricen, die der Verwachsung und Spaltung, feindlich gegenüberstanden, sind in nenerer Zeit noch drei Theorieen hinzngekommen, von deneu die älteste, dic Rauber'sche Radiationstheorie, die Entstehung der Doppelmonstra von einem einheitlichen Gesichtspunkt beurtheilt, während die beiden neneren, die Gerlach'sche Bifurkationstleerie und die Klaussnersche Theorie der Fission mit Postgeneration, die Einheit des Princips aufgebend, nur auf eine bestimmte Form der Doppelbildungen sich beziehen. Eine genalue Darstellung der im Lanf der Zeit von den versehiedenen Forschern über die Natur der Doppelbildungen geäusserten Ansichten findet sich in Rauber's Abhandlung über die Theorieen der excessiven Monstra (Virchow's Arehiv, Bd. 71) und in Leo Gerlach's Monographie über die Entstehungsweise der Doppelbildungen; ich kam mich daher auf eine kurze Skizziruug der verschiedenen Theorieen beschränken.

Die älteste Theorie ist die Verwachsungstheorie, welche in ibrer ursprünglichen Fassung die Doppelbildungen aus der Verwachsung zweier anf verschiedenen Eiern entstandener Embryonen herleitet. Alle Beobachtungen an jungen Doppelbildnngen, besonders aber die Befunde Panum's an Huhnereiern mit doppeltem Dotter haben gelehrt, dass eine solche Verwachsung: unmöglich ist, dass vielmehr alle Doppelbildungen aus einem Ej 
resp. auf einem Dotter entstehen. Die Verwachsungstheorie hat daher im Lauf der Zeit eine andere Fassung bekommen, in welcher sie besonders von Lereboullet, B. Schultze, Dareste und $P$ anum vertheidigt worden ist: Nicht zwei anf verschiedenen Eiern entstandene Embryonen, sondern zwei in demselben Ei entstandene, ursprïnglich gresonderte Embryonalanlagen sollten sich im Lauf der Entwickelung nähern und gan\% odler theilweise mit einander verschmelzen.

Aber anch in dieser Form hat die Verwachsungsthenrie in den letzten Jahren keinen Vertheidiger mehr gefunden. Vermag sie doch durchaus nicht $z$ n erklären, warum in allen hisher beobachteten Fällen stets genan identische Theile beider Embryonen mit einander verbunden sind. Theoretiseh lässt sich ferner einwenden, dass dic Verwachsung doch nur eine sekundäre Erscheinung ist, dic wohl einen innigeren Zusammenhang beider Komponenten herbeiführen kann; von ciner ursprünglichen Entstehung der Doppelbildungen durch Verwachsung kann aber nicht die Rede sein, da die Komponenten schon vom Infangr an dadurch, dass sie in demselben Fruchthof angelegt werden, kontintirlich mit einander verbunden sind.

Mehr Anhänger hat ihre Gegnerin, dir spaltungstheorie, aufzuweisen, darunter eine grosse Anzahl der bedentendsten Embryologen, z. B. C. F. Wolft, J. F. Meckel, v. Baer, Joh. Wïller, Valentin, Bisch off, Leuckart, Reichert nnd andere. In seinem grossen Werke iiber die Missbildungen des Mensehen hat Ahlfeldt. der jüngste Vertreter dieser Theorie, dieselbe scharf formulirt. Er stellt sich vor, dass Mehrfachbildungen und eineiige Zwillinge aus einem ganz normalen $\mathrm{Ei}$ entstehen, dessen Befruchtung und Furchung in der gewöhnlichen Weise abläuft. Nach der liurchung, jedoch bevor die ersten Spuren des Embryo auftreten, wird die aus indifferenten Furchungskngeln bestehende Zellmasse durch den Druck der zu engen Zona pelluci d a mehr oder weniger tief gespalten. Durch den fortdauernden Drnck der Zona pellucida werden beide Hälften auseinandergetrieben, wollurch eine Drehung der mittlerweile in jeder Hälfte entstandenen Embryonalanlagen stattfindet. Die Spaltung findet immer in cler Lüngsachse statt.

Die Annahme einer Spaltung der Furchungszellmasse durch den Druck Rer Zona pellucida stïtzt sich lediglich auf Speku- 
lationen und Vermuthungen, und mehrere thatsächliche Beobachtungen sprechen dagegen. Panum beobachtete nämlich mehrere Hühnereier mit strangförmiger Verdickmug der Dotterhaut, die quer über den Keim hinweglief und eine tiefe Einschnürung veranlasste, aber trotzdem die Entwicklung eines einfachen normalen Embryo nicht gestört hatte. Feruer kann ich ni cht zugeben, dass es bisher gehungen wäre, durch irgend welche mechanische Eingriffe Doppelbildungen zu erzeugen; alle derartigen Behatptungen laben sich als irrthümlich erwiesen und sind von Schrohe und anderen widerlegt worden. Wenn Ahlfeldt endlich annimmt, dass die nach der Furchung entstandenen Zellen noch ind ifferent seien, so ist das ein Irrthum; denn jede Furchungszelle hat nach den Beobachtungen Roux' am Froschei ihre besondere Bedeutung. Daher mïsste Ahlfeldt zugleich annehmen, dass jedle der durch Spaltung entstandenen Hälften sich mehr oder weniger an einem vollständigen Embryo regeneriren könne.

Diesen beiden Theorieen hat vor 15 Jahren $R$ a uber als eine dritte seine $\mathrm{R}$ adiationsthe orie gegenübergestellt. Er geht von der Ammahme aus, dass bei der normalen Entwicklung der Knochentische nach geschehener Furchung auf der aus einem dïnnen Mittelfeld und dem stärkeren Rumlwulst bestehenden Keimscheibe die Kopfanlage als ein centripetaler Vorstoss des Randwulstes erscheint, während der übrige Körper durel suceessive Aneinamlerlagernng der beiden Randwulsthälften gebildet wird. Gestiitzt anf die Beobachtung zahlreicher, zum Theil sehr junger Mehrfachbildungen von Knochentischen behauptet er nun, dass die Anlage einer Doppelbildung dadurch geschehe, dass statt eines Vorstosses $z$ w e i radiü gegen das Centrum der Keimscheibe gerichtete Ausstrahlungen des Randwulstes erscheinen. Nur wenn beide vorderen Embryonalanlagen sich in Oppositionsstellung befinden, d. h. wenn ibre Achsen unter einem Winkel von $180^{\circ} \mathrm{zu}$ einander eingestellt sind, werden die jeder von beiden zngehörigen Randwulststreeken gleich sein und zwei getrennte Embryonen entstehen können. In allen übrigen Fällen werden die beiden vorderen Embryonalanlagen den Randwulst in zwei mugleiche Theile zerlegen, eine kürzere „innere Zwischenstrecke" und eine längere näussere Zwischenstrecke". Von der inneren Zwischenstrecke werden die medialen einander 
zugrekehrten Körperhälften gebildet, während aus der äusseren Zwischenstrecke die lateralen und schliesslich der gemeinsame Hinterleib hervorgehen. Auch beim Hühnchen entsteht nach $\mathrm{R}$ a u b e r's Auffassung der vordere Theil des Primitivstreifens als ein Vorstoss des Keimwulstes, der hintere wird durch Conjunction allerdings nur der in nächster Nähe liegenden Theile des Keimwulstes geliefert, während der bei weiten grö̈ssere Abschnitt des Keimwulstes lediglich der Dotterumwachsung dient. Durch diesen zwischen Hühnchen und Knochenfischen bestehenclen Untersehied erklärt es sich nach Rauber's Angabe leicht, warum bei ersteren die Anakatadidyni und Katadidymi, bei letzteren dagegen die Inadidymi bei weitem überwiegen.

Die Rauber'sche Radiationstheorie hat gleichfalls inre Beleutung verloren, seitdem man fast allgemein die Ansicht anfgregeben hat, dass der Embryo aus dem Randwulst durch Aneinanderlagerung seiner beiden Hälften entstehe. Der Randwulst dient vielmehr auch bei Fischen zum grössten Theil der Dottersackbild ung, nur der der Embryonalanlage nahe gelegene Theil, die Ungebung der Randkerbe, kam zum Aufbau des Embryo beitragun. Beim Hühnchen filsst $R$ auber den vorderen Theil des Primitivstreifens als Kopfanlage anf, während doch feststeht, dass die Medullarwülste sich vor dem Primitivstreifen anlegen.

Vor 10 Jahren hat Leo Gerlach in seinem umfangreichen Werke über die Entstehungsweise der Doppelmissbildungen bei len höheren Wirbelthieren, in welchem sämmtliche bisher beobachtete Mehrtachbildungen des Hühnchens zusammengestellt worden sind, die Rauber'sche Theorie für die Knochentischdoppelbildungen und die meisten Doppelbildungen der übrigen Wirbelthiere im Grossen und Ganzen anerkannt; nur die seltenen An a d idy mi des Hühmchens scheinen ihm nach derselben nicht erklärt werden zu können. Um über die Entstehnng der letzteren Klarheit zu gewinnen, versuchte er dieselbon künstli e h herzustellen, indem er die Eier vor der Bebritung bis auf eine kleine über der Keimscheibe gelegene Y-förmige Stelle mit Firniss überzog, so dass die vorderen Schenkel des $Y$ dem künftigen Kopfende des Embryo entsprachen. Dadurch, hoffte er, würde der hinten einfach angelegte Primitivstreif bei seinem nach vorn gerichteten Wachsthum sich am vorderen Ende gabeln, weil die zu seiner Entstehung nothwendige lebhafte Zellwucherung 
von der Sanerstoffzutuhr abhängig sei. Unter 60 auf diese Weise behandelten Eiern zeigten allerdings nur zwei eine nach Gerla ch's Ansicht unbestreitbare vordere Verdoppelung. Auf diese experimentellen Ergebnisse gestützt hat er seine Bifurkationstheorie anfgestellt, nach welcher die Inadidymi des Hühnchens dadurch entstehen, dass nur eine einzige Embryonalanlage in die Area pellucida einstrahlt, welche in ihrer weiteren nach vorn gerichteten Ausbildung (vielleicht durch Verdickmgen der Dotterhant) bald frither, bald später die Medianlinie verlässt, $\mathrm{nm}$ gabelig divergirend in zwei Schenkel ausznlaufen.

Die experimentellen Ergebnisse, auf welche Gerlach sich stïtzt, kann ich nicht fïr beweisend erachten. Die beiden angeblich dabei erzielten vorteren Verdoppelungen sind den Abbildungen und der Besehreibung nach nicht mit Sicherheit als solehe zu crkennen. Im ersten Fall handelt es sich nm einen verbreiterten, nicht scharf begrenzten Primitivstreifen, der an seinem vorderen Ende ¿ kuze tivergirende Fortsiitze trägt und in einem o Tage lang bebriiteten Ei gefunden wurde. Dergleichen Unregelmässigkeiten in der Bildung des Primitivstreifs kommen in Eiern aus der Brtitmaschine ïfters vor; zum mindesten hätte Gerlach jene beirlen Ansläufer auf Schnitten untersuchen miissen, um iiber ihre Natur sichere Angaben machen zu können, zumal da eine Rinne noch nicht vorhanden war. Der wweite Fall betrifft ein schom ziemlich weit entwickeltes Hühnchen mit sehr breiten Kopf, an welchem von der Dorsalseite her 2 durch einen tiefen Einsclinitt zretrennte Hirnanlagen zu erkenneu wareu. Auch dicser Embryo ist anf Schnitten nicht untersueht worden; es bleibt daher zweifelhaft, ob es sich nicht bloss an eine Henmungsbildung handelt, bei welcher beide Hedullarfalten sich nicht vereinigt haben.

Aber auch theoretische Griinde veranlassen mich, die Gerlach'sehe. Theorie zu verwerfen. Zunächst ist die ganze Vorstellung Gerlach's, dass durch Beschränkung der Sanerstoffzufuhr auf eine Y-törmige Stelle, deren Schenkel $1 / 2-1 \mathrm{~cm}$ breit sind, der Primitivstreif sich vorn gabeln müsse, etwas absonderlich, dem der Sanerstoff wird sich in Eiweiss auch in weiterer Umgebung verbreiten kömnen; terner aber ist die Grösse des Prinitivstreif's im Verhältniss zn jener Y-förmigen Figur so gering, dass zu allen seinen Theilen befurm Sancrstoff hin- 
zutreten kann. Die sauerstoffzufuhr ist nur im ganzen herab. gesetzt; daraus erklären sich leicht die vielen einfachen Missbildnngen, die Gerlach erhielt. Ausserdem ist die Voraussetzung: Gerlach's, dass der Primitivstreif von hiuten nach vorn in die Area pellucida eimwachse, umrichtig, da mach den genauen Untersuchungen Duval's nicht der hintere, sondern der vordere Theil des Primitivstreifens znerst entsteht. Mithin kann von ciner Bifurkation eines mspriunglich einfachen Primitivstreifens nicht die Rede sein.

Vor 2 Jahren ist wiederum eine tumfangreichere Arbeit uber Mehrfachbildungen bei Wirbelthieren von F. Klanssner erschienen, in welcher uahezu $50 \mathrm{zmm}$ Theil sehr junge Mehrfachbildungen aus sïnmuthichen Wirbelthierklassen beschrieben und auf 12 Tafeln alggebildet worden sind. Was die Genese derselben betrifft, so unterseheilet Klaussner 2 Gruppen ron Mehrfachbildmgen, solehe, die durch primäre Pluralität der Anlage und solche, die durch Fission mit Postgeneration entstehen. Die erstern kommen dadurch zustande, dass vom Anfang rer Entwicklnng an 2 oder mehrere selbständige Embryonalanlagen auftreten, welche entsprechend den in der Radiationstheorie entwickelten Grundsüt\%en verschieden zu einander gelagert sein kömmen, nicht aber sich mit einander vereinen. Die zweite (iruppe entsteht auf dem Woge der Spaltung, jedoch nicht in cler Weise, dass jede der beiden Fälften die Fähigkeit hat, sich selbständig zu rollständigen Embryonalanlagen zu entwickeln, sondern so, dass jede Hälfte die ibr fehlende Hailfte durch Postgeneration ans den Nachbarmaterial erzeugt. Der Begriff Postgeneration stammt von Roux. Dieser Forscher fand nämlich, wenn er am Froschei mit einer glühenden Nadel eine der beiden ersten Furchungskngeln zerstörte, dass die andere sich in gewöhnlicher Weise zu einem im wesentlichen normalen halben Embryo entwickelte. Die durch den Stich ibrer Entwicklungsfähigkeit beraubte Furchungszelle konnte wieder belebt werden durch Reorganisation, Nucleisation und Cellulation, d. h. dadurch, dass vom freien Seitenrand jedes Keimblattes (Unterbrechungsfläche) eine Anzahl Kerne in den Dotter einwanderten und darin eine zur Entstehung der fehlenden Embryonalhälfte führende Zellahgliederung: veranlassten.

Wir werden bei der Beurtheilung der Klaussner'schen 
Theorie wohl dem Umstand Rechnung tragen müssen, dass die Roux'sche Arbeit kurz zuvor erschienen war, und die Abfassung der Untersuchungen von Klaussner in die Zeit der litterarischen Einwirkung der Roux'schen Mittheilungen fiel. Eine thatsächliche Begründung fehlt der Klanssner'schen Annahme gänzlich, da weder für das Zustandekommen einer mechanischen Spaltung, noeh einer Postgeneration beweisende Benhachtungen vorliegen. Wenn auch Roux nachgewiesen hat, dass eine fiur den Moment abgetödtete Furchungskugel des Froscheies durch Einwanderung von Kernen aus der sich weiter entwickelnden andern Furchungszelle wieder belebt werden kann, wodurch der anfangs halbe Embryo zul einem ganzen sich erzäzzt, so dart man doch daraus nicht schliessen, dass nach spaltung einer Furchungszellmasse jedle Hälfte durch Postgeneration einen ganzen Embryo erzeugt; demn es fehlt ja der abgetioltete Dotter, welcher in jenem Falle wieder belebt und organisirt wirl, und eine Kerneinwanderung in den Dotter ist nicht so unmittellar möglich. da die subgerminale Höhle Keim und Dotter trennt.

Aus dieser kurzen Zusammenfassung sneht zur Genüge hervor, wie sehr die Ansichten der verschiedenen Autoren noch von einander abweichen. Eine Einigung ist nur dadurch zu erreichen, dass durch Beschreibung möglichst junger typischer Doppelmonstra eine melr gesicherte Grundlage für die Beurtheilung. ihrer Entstehung geschaffen wird. Aus diesem Grunde soll im folgenden eine junge Doppelbildung des Hühnchens beschrieben werden, welche ich im Sommer 1891 beobachtet habe, und die besonders deshalb ron grossem Werth ist, weil sie ein sehr junges Stadium der so seltenen Duplieitas anterior vorstellt.

\section{Beschreibung des Falles.}

Bevor ich zur Beschreibung der von mir beobachteten Doppelbildung ïbergehe, will ich kur die Methode schildern, welche mir in diesem Falle wie auch sonst zur genauen Untersnchung ron Hühnerkeimscheiben diente.

\section{a. Methode.}

Nach sorgfïltiger Eröffnung des Eies und möglichster Entfermung des Eiweisses wird der Dotter in $10 \%$ Salpetersäure 
gebracht, in welcher er ungefaihr 10 Minuten bleibt. Alsdann wird mit einer feinen Scheere die Dotterhaut rings um die Keimscheibe umsehnitten, und durch geringe Bewegungen der Flüssigkeit z. B. mittels eines Spatels die letztere sowohl vom Dottęr als auch von der ihr auflegenden Dotterhaut befreit. Vor allen anderen von mir versuchten Fixirungsmitteln hat die $10 \%$ Salpetersäure den grossen Vorzug, dass sie die Ablösung der Keimscheibe vom Dotter und von der Dotterhaut sehr erleichtert und zugleich der Keimscheibe eine so vorzügliche Consistenz giebt, dass dic wïhrend der weiteren Manipulationen unumgünglichen Verbiegungen ohne den geringsten Schaden ertragen werden. Die abgelöste Keimscheibe wird dann mit einen breiten Hornspatel in ein Gefäss mit planem Boden gebracht, welches wiederum etwas $10 \%$ Salpetersäure enthält. Hierauf wird mit einer Pipette die Salpetersïure abgesogen, so dass sich die Keimscheibe glatt anf den Boden des Schälchens legt, und nun wird anfangs tropfenweise $2 \%$ Alaunlösung zugesetzt. Sofortiges Uebertragen aus der Salpetersäure in die Alaunlösung, sowie ans dieser in Alkohol, der zur weiteren Härtung dient, führt leicht zu störenden Verbiegungen der Keimscheibe. Nach der Härtung in allmählich verstärktem Alkohol können Boraxkarmin, Bühmer'sches Hämatoxylin oder Alauncochenille verwandt werden, in diesem Falle wurde letztere benutzt.

Dic so vorbereitete Keinscheibe wird in Nelkenöl aufgehellt und dann mit Hülfe des Hartnack'schen Embryographen bei 20 facher Vergrösserung genau gezeichnet. Die Einbettung geschieht in Paraffin. Die Keimscheibe ist rom Schwanzende her in durchweg $0,02 \mathrm{~mm}$ dicke Schnitte zerlegt worden, welche der Reihe nach mit Nelkenölcollodium auf den Objectträger aufgeklebt wurden. Man sieht also auf ihre dem Schwanzende zugekehrte Seite. Die Schnittrichtung zur Medianachse der Doppelbildung, worunter ich eine den Divergenzwinkel der Chorden halbirende Linie verstehe, ist eine fast genau senkrechte.

Auf die Area pellucida kommen 178 Schnitte von $0,02 \mathrm{~mm}$ Dicke; daraus lässt sich die Länge derselben $=3,56 \mathrm{~mm}$ berechnen. Nun ergibt sich durch Messung an der genau $20 \mathrm{mal}$ vergrösserten Flächenzeichnung; dass der helle Fruchthof vor der Paraffineinbettung (während des Aufenthalts der Keimscbeibe in Nelkenöl) eine Länge ron $4,4 \mathrm{~mm}$ besass. Darans geht hervor, 
dass die zarte Keimhaut infolge der Paraffineinbettung eine sehr beträchtliche Verkleinerung erfahren hat, und zwar um $19 \%$ der ursprüngliehen Länge. Weitere genaule Messungen an dem Flächenbilde einerseits und Berechnungen aus ler Schnittzahl andererseits lehrten, dass nicht alle Theile des Blastoderms vou der Schrumpfung in gleicher Weise betroffen wurden, vielmehr stellte es sich heraus, dass die Verkürzung innerhalb des Embryonalkörpers eine geringere war als die der Irea pellncirla. Ja selbst die Verkürzung des Embryonalkörpers ist keine ganz gleichmässige, sondern die massigeren Parthieen des Kopfes sind verhältnissmässig weniger. (um $12 \%$ ) verküurt, als die des Uittelkörpers und Schwanzeides $(16,9 \%)$.

Will man also im Flächenbilde den ()rt feststellen, der einem bestimmten Schnitt entspricht, so muss man diese. $Y$ erschiedenheiten in der Schrumpfung kennen und berücksichtigen, da sie doch so beträchtlich sind, dass sie zu fehlerhaften Bestinumungen und falschen Schlïssen fïhren kömen.

\section{b. Flächenbild.}

Das Ei, welches weder in Bezug auf scine Grösse noch auf seine Form etwas Abnormes darbot, wurde 36 stunden lang im Brïtofen bei ungefähr $39^{\circ} \mathrm{C}$. bebriitet, entsprach jedoch seiner Entwicklung nach nur einem 24 Stunden bebrüteten. Der Rand der Keimhant hatte den Aequator der Dotterkugel noch nicht erreicht. Der Dotterhof war von gewöhnlicher Beschaffenheit. Der Gefässhof war nach aussen noch nicht iiberall scharf begrenzt. Seine Form entsprach einem Ovoid mit rorn gelegenem spitzen Pol; in der Länge mass er $5,3 \mathrm{~mm}$, in der Breite $4,7 \mathrm{~mm}$ : Die Anlage des Sinus terminalis war noch nicht recht deutlich. Blutinseln waren im Gefässhof schon vorhanden.

In Figur 1, welche die Keimscheibe bei durchfallendem Licht in der Rückenansicht 20 mal vergrössert darstellt, fallt zunächst die e igenth i m li ehe Form der Area pellucida auf. Sie ist zwar wie in der Norm exquisit birnformig;, indessen entspricht ihr breiterer Theil nicht wie gewöhnlich dem Kopfende, sondern dem Schwanzende des Embryo. In dieser Area pellucida liegt dem linken Rand beträchtlich näher als dem rechten cine kurze, aber sehr breite Embryonalanlage, die an dem Verhalten der Chordae leicht als eine Duplicitas anterior erkannt werden kann. 
In ihrem mittleren Theil treten nämlich deutlich 2 als Chordae aufzufassende Stränge hervor, die unter einem sehr kleinen Winkel divergiren, hinten aber zu einem gemeinsamen Strang zusammentreten, der nach kur\%en Verlauf in eine rundliche Anschwellung zu endigen scheint. Von dieser letzteren, über welcher eine Rinne nicht zu erkennen ist, geht schräg nach rechts hinten ein nicht scharf' begrenzter, mit einer deutlichen Rinne versehener Streifen aus, über dessen Deutung als Primitivstreif wohl kein Zweifel bestehen kann. Von ibm strablen nach beiden Seiten 2 bandartige Schattirungen, anscheinend Verdickungen des Mesoderms ans, die rechte schmal, im weiteren Verlauf breiter werdend und weiter nach dem Kopfende reichend als die breite kürzere linke. Diesen Bündern entsprechende Rimnen konnten nirgends mit Sicherheit erkannt werden. Den Enden dieser beiden Streifen entsprechen die eigenthümlichen ausgedehnten Verbreiterungen des hellen Fruchthofs.

Ungefähr in der Mitte der Embryonalanlage tinden sich 3 oder besser gesagt $4 \mathrm{R}$ e i h e $\mathrm{n}$ dunkel gefärbter Körper, welche ihrer Gestalt und Anordnting nach als $\mathrm{Urw}$ irbel aufufassen sind. Lateral von der linken Chorda liegen 3 deutlich begrenzte Urwirbel von gewöhnlicher Form, lateral von der rechten Chorda sind ebentalls. 3, aber unregelmässig gestaltete Urwirbel vorhanden, der vordere gross, der mittlere sehr schmal, der hintere anscheinend ans 2 Stücken bestehend. Die in der Mitte gelegene Reihe zeichnet sich durch grössere Breite der Urwirbel aus; indessen lassen sich bei stärkerer Vergrösserung 2 neben einander gelegene Reihen von Urwirbeln erkennen, in Grösse und Form beiderseits nicht ganz gleich.

An diese 3 Urwirbelreihen schliessen sich hinten 3 Urwirbelplatten an; die beiden lateralen verlaufen in gewöhnlicher Weise neben den Chorden, die mittlere von der Gestalt eines gleichsehenkligen Dreiecks, dessen Spitze bis zur Theilungsstelle der Chorda reicht, lässt von einer Trennung in 2 symmetrische Hälften nichts erkennen.

\section{c. Beschreibung der Schnitte.}

Um ïber den Zusammenhang der beiden Componenten des Doppelmonstrums und die Verëndermngen in der Anlage der Primitivorgane Aufschluss zu geben, sind 10 vom Kopf zum Archiv f. mikrosk. Anat. Bd. 41 
Schwanzende hin auf einander folgende Schnitte bei 50 -facher Vergrösserung in den Figuren 2-11 abgebilitet worden. Die jedem dieser Schnitte in Figur 1 entsprechende Stelle ist durch einen Strich möglichst genau angegeben worden.

Aus der Betrachtung der Schnitte ergiht sich nun fiolgendes :

Die Abschniirung des Kopfes von der Keimhaut ist noch nicht weit vorgeschritten, erst bis zu einer Entfernung vou 0,14 mm vom Kopfende, d. h. von ter Kuppe des den Kopf vorn begrenzenden Ectoderms.

Die rechte If edullarinne crscheint als ganz Hache Furche 0,08 mm rom Kopfende entfernt; auf den folgenden Schnitten nimmt sie noch an Tiefe zt, ist aber so schmal, dass ihr Lumen dureliweg fast strichfömig erscheint. Sie steht von Anfang an nicht ganz senkrecht zum Ectoderm, sondern ist mit ihrem Grund lateralwärts gerichtet, und zwar um so mehr, je weiter sie sich vom Kopfende entfernt.

Links heginnt $0,2 \mathrm{~mm}$ rom Kopfende entfernt im Ectoderm eine Rinne deutlich zu werden, welche als solche in einer Länge von $0,1 \mathrm{~mm}$ bestehen bleibt, um dann allmählich in eine solide unregelmässig kegelfömige Ectodermmasse iberzngehen, welche anfangs (vgl. Fig. 3) kein deutliches Lumen erkennen lässt, später aber der rechten Medullarrinne an Ciestalt gleich wird. Die ganze Bildung halte ich für die linke Medullarrinne, deren Lumen auf einigen Schnitten wegen ihrer bedentenden Krümmung nach links hin nicht deutlich ist.

Zwischen beiden Hirnanlagen ist weiter vorn eine ziemlich breite sie verbindende Ectodermbrücke vorhanden, die nach dem Schwanzende hin immer schmäler wird. Schliesslich (Fig. t) stossen die medialen Lamellen beider Medularrinnen hart aneinander und erscheinen damn wie ein Keil mit abgerundeter Spitze, der weiter hinten immer kleiner und niedriger wird, um $0,56 \mathrm{~mm}$ vom Kopfende entfernt ganz zu verschwinden. Damit ist es zur Bildung jener schon im Flächenbilde so auffallenden, weit offenen gemeinsamen Medullarrinne gekommen, welche 3 anfangs etwas nach innen übergeneigte, später senkrecht zu einander stehende Wände besitzt, eine untere beiden Componenten gemeinsame $(0,24 \mathrm{~mm}$ breit) und 2 seitliche von betriichtlicher Höhe $(0,2 \mathrm{~mm})$. Während das Ectoderm weiter vorn mit scharfer 
Biegung unter einem rechten Winkel in die Medullarwände sich fortsetzt, wird dieser Winkel nach hinten allmählich stumpfer, die Knickung geringer, so dass die Seitenwände der Medullarrime dadurch schriag von aussen nach innen abfallen. So bekommt dic Medullarrinne ein immer weiter klaffendes, aber auch Hacheres Lumen. Allmählich erhebt sich weiter hinten in der Medianlinie das Ectoderm in Form eines kleinen Buckels, der wohl durch das darunter sich entwickelnde, zwischen beiden Chorden eingeengte Mesoderm verursacht ist. Daher sieht man ungeführ an der Stelle der Chordenspaltung 2 nüher an einander gelegene etwas tiefere Rinnen, welche sich im weiteren Verlauf schnell abflachen und endlich, 1,7 mm vom Kopfende entfernt, verschwinden.

Der Vorderdarm ist eben angelegt und noch sehr kurz, aber von betrichthlicher Breite. Auch er ist beiden Embryonen gremeinsam, nur 2 ganz geringe Vorwölbungen seiner Wand nach links und rechts vorn, von welchen die rechte weiter vorwärts reicht als die linke, kömen als Andeutung einer doppelten Anlage angesehen werden. Dementsprechend erseheint, wenn man die Schuittreihe vom Kopf nach dem Schwanz hin durchmustert, das Lumen zunächst im rechten Abschnitt des Kopfes, um sich dann allmählich nach links weiter auszndehnen. Ist das geschehen, so erscheint der Vorderdarm als ein querer den Kopf durchsetzender breiter Spalt, dessen laterale Partieen zwischen ler Medullaranlage und dem Ectoderm der Seitenrand des Kopfes nach oben emporstreben; seine obere Wand ist dünner als die untere und in der Medianlinie nach oben vorgebuchtet. In einer Entfernung von $0,22 \mathrm{~mm}$ vom Kopfende verschwindet seine untere Want. Die lateral von den Medullaranlagen gelegenen Vorbuchtungen des Entoderms bleiben noch eine Zeit lang bestehen; die mediale Vorwölbung des Entoderms nach oben wird zunächst deutlicher, um dann allmählich zu verschwinden und schliesslich kurz vor der Urwirbelgegend in eine gegen den Dotter gerichtete Vorbuchtung: des Entoderms ïberzugehen, welche durch die stark wuchernde, «wischen beiden Chorden eingeengte Mesodermmasse veranlasst ist. Hinter den Urwirbeln erscheint das Entoderm immer mehr als eine plane, unter dem Embryo einherziehende Lamelle.

Die Chorden reichen sehr weit nach vorn und kommen 
daher, wenn man die Serie von vorn nach hinten durcbsieht, schon sehr früh zu Gesicht, erst die rechte, wenige Schnitte weiter nach linten anch die linke. Die rechte Chorda erscheint in ihrem vordersten Abschnitt als eine dichtere Anhüufung von Mesodermzellen, die aber vom Entoderm absolut nicht zu trennen sind und auch gegen das umliegende Mesoderm nicht scharf abgegrenzt sind. Die linke Chorda erscheint in ihrem vordersten Theil als Verdickung des Entoderms, wenige Schnitte weiter hinten verhält sie sich wie die rechte. Beide Chorden liegen immer unter dem Fundus der zugehörigen Medullarrinne und grenzen sich weiter hinten schärfer gegen das übrige Mesoderm $\mathrm{ab}$, auch das Entoderm zieht dann als cin Strang . spindelförmiger Zellen unter ilmen fort. Nach der Vereinigung beider Medullarrinnen liegen sie an den unteren Kanten der breiten tiefen Rinne, wie Fig. 6 deutlich zeigt. Vorn breiter und niedriger wird ihr Durchschnitt hinten allmählich höher und schmäler, von der Form eines sehr langen Ovals. Wenn dann die Medullarrinne sich abgeflacht hat, werden sie bedeutend voluminöser, und zwar ist die rechte Chorda stärker als die linke. Endlich erscheinen sic mit dem Ectoderm verschmolzen, um dann, nachdem das zwischenliegende Mesorlerm immer geringer geworden ist, zu einer Chordenmasse sich zu vereinigen, die weder vom Ectoderm noch vom Entoderm zu trennen ist.

Was die Urwirbel betrifft, so lassen sich auf den Schnitten leicht 4 Reihen unterscheiden, 2 mediale und 2 laterale. Die letzteren besitzen die gewöhnliche Form und Beschaffenheit, nur der dritte rechte Urwirbel zeigt auf dem Durchschnitt eine Verdünnung seiner Substanz in der Mitte, weshalb er auf dem Flächenbilde zerklüftet erscheint. Die medialen Urwirbel beginnen etwas weiter vorn als die lateralen und sind ebenfalls $z u$ je 3 vorhanden; die vorderen sind gleich gross, von den mittleren ist der linke breiter als der rechte, die hinteren sind wieder ziemlich gleich.

Nach vorn von den Urwirbeln ist das Mesoderm zwischen beiden Chorden stark gewuchert und hat das Entoderm wie einen breiten Kiel gegen den Dotter vorgetrieben. Die lateralen Mesodermplatten sind sehr stark ausgebildet und von bedeutender Dicke, besonders die linke, welche auch noch in der Urwirbelgegend weit stärker bleibt als die rechte. 
Hinten schliessen sich an dic Urwirbel 3 Urwirbelplatten an, 1 mediale und 2 laterale. Die erstere hat auf dem Durchschnitt zuerst die Form einer Ellipse, um weiter hinten allmählich mehr kreisförmig zu werden. Endlich wird sie immer kleiner und verschwindet schliesslich vor der Vereinigungsstelle beider Chorden; von der Ungebung ist sie immmer scharf abgegrenzt. Die lateralen Urwirbelplatten zeigen nichts Besonderes.

Im vorderen Bereich des Embryo ist die Leibesh $\ddot{b}$ h le beiderseits schon angelegt. Auf der rechten Seite reicht sie etwas weiter nach vorn als der Kopf des Embryo; dort beginnt sie als kleiner Spalt, der nach hinten schnell an Grösse zunimmt und za einer grossen Höhle wird, deren obere Wand das Ectoderm nach oben kuppelförmig vortreibt. Links beginnt eine Höhle erst $0,3 \mathrm{~mm}$ hinter dem Kopfende und erreieht nirgends die Ansdehmung der reehts gelegenen. In der Urwirbelgegend werden beide Leibeshöhlen allmählich kleiner; statt einer einheitlichen Höhle erscheinen dann nur noch mehrere kleine Hesodermspalten, welche sich hinter den Urwirbeln allmählich verlieren.

\section{d. Rekonstruction.}

Sehr schwierig ist es, sich aus der blossen Betrachtung der Serie ein klares Bild über den Hinterkürper des Embryo zu machen. Um über den Verlanf der Primitivrinne und die Natur jener oben beschriebenen sichelförmigen Ausstrahlungen des Primitivstreifens Klarheit zu gewinnen, habe ich eine Flächenrekonstruction der hinteren Embryonalgegend gemacht, welche in Fig. 12 wiedergegeben worden ist. Es wurde dazu das käufliche Millimeterpapier benutzt, welches mit einer recht genatuen mm-Quadrirung versehen ist. Die Rekonstruction wurde bei 50 facher Vergrösserung ausgeführt, bei welcher die Dicke eines jeden Schnittes $1 \mathrm{~mm}$ entspricht. Nunmehr war es also nur nöthig, auf jeder folgenden Horizontallinie die gewünschten Punkte jedes folgenden Schnittes aufauzeichnen. So wurden die Grenzen der Area pellucida, die im Ectoderm vorhandenen Rinuen, die Grenzen der Chorden (letztere als rothe Linien) eingetragen. Die Horizontalstriche bedeuten weiter vorn die Medullarplatte, weiter hinten die Stellen des Ectoderms, an welchen eine Verbindung desselben mit dem verdickten Mesoderm stattfindet. Die beige- 
fïgten Ziffern sagen von jedem Schnitt aus, der wic vielte er rom Kopfende aus ist.

Die Form der Area pellucida, welche so gewommen wurde, stimmt, wie man sieht, gut mit der des Fliichenbildes ïberein. Die Medullarrinnen liegen immer ziemlich genau über den Chorden und convergiren ein wenig nach hinten. Kurz hinter der Vercinigungsstelle beider Chorden erreichen sie ilr Ende, nachdem sie vorher allmählich flacher geworden sind.

Die Chorden, von welchen die rechte hier deutlich stärker erscheint als die linke, gehen hinten in einen einzigen breiten Strang: iiber, wcleher aus dicht an einander liegenden Mesodermzellen besteht, die weder vom Ectoderm noch vom Entoderm sich abgrenzen lassen. Dadurch, dass dieser gemeinsame Chordenstrang weiter hinten breiter wird, entsteht jene im Flächenbild so dentlich hervortretende knotenförmige $\mathrm{An}$ schwellung. Zwischen dem Mesoderm dieses Stranges und dem seitlich gelegeneu Hesoderm besteht nur der Unterschied, dass im ersteren die Zellen viel dichter liegen, woraus eine dnnklere Färbung resultirt, und dass sie mit den beiden anderen Keimblaittern continnirlich verbunden sind, was in dem seitlichen Mesoderm nicht der Fall ist. Weiter hinten wird dieser Unterschied immer undeutlicher, so dass endlich eine bestimmte Gren\%e sich nicht mehr feststellen lässt.

Nachdem beide Medullarrinnen kurz hinter der Stelle der Ghordenvereinigung; nämlich auf' Schnitt $86(1,72 \mathrm{~mm}$ voln Kopfende ans gerechnet) ilır Ende gefunden haben, treten im Schnitt 88 (also $0,04 \mathrm{~mm}$ weiter hinten) $\mathrm{z} w \mathrm{i}$ sehr flache Rinnen auf, von denen die linke ctwas tiefer ist als die rechte. Auf den folgenden Schnitten nähern sie sich allmählich, um endlich auf Schnitt 94 (entsprechend einer Entfernung von $1,88 \mathrm{~mm}$ ) $\mathrm{zu}$ einer Primitivinne sich zu vereinigen. Diese verläuft anfangs gerade nach hinten, später aber (von Schnitt 102 an) schräg rechts und endet auf Schnitt 113 (2,26 mm vom Koptende entfernt). Auf einigen Schnitten (106 und 107) erscheint die Primitivrinne wieder doppelt, indessen ist es möglich, dass es sich hier nur um eine künstliche Faltung des Ectoderms handelt.

Die seitlichen Anhänge des Primitivstreifens treten in Fig. 12 in ihrer Form sehr deutlich hervor. Der rechte 
ist lang und schmal und reicht viel weiter nach vorn als der linke, welcher kurz und breit erseheint. Am vorderen Ende des rechten Streifens findet sich eine $0,05 \mathrm{~mm}$ lange $\mathrm{R}$ in $\mathrm{n}$ e, welche in Fig. 11 wiedergegeben ist. Auch im linken Streif ist das Ectoderm nicht glatt, sondern zeigt allerlei Biegungen und Vertiefungen, jedoch liess sich eine fortlaufende Rinne nicht auffinden. Auf den Sehnitten zeigt es sich, dass der rechte Streif gebildet wird durch eine Verlöthung des Ectoderms mit dem Mesoderm, welche beide verdickt sind; letzteres ist aber nur am vorderen Ende des Streifens deutlich verdickt, sonst nur dichter als in der Umgebung. Der linke Streif besteht aus verdicktem Mesoderm, das mit dem Ectoderm in dem in der Rekonstruction gezeichneten Umfang zusammenhängt und ein dichteres Gefüge bat als das übrige Mesoderm. Ungeführ anf dem 100sten Schnitt (2,0 mm vom Kopfende entfernt) confluiren erst der linke, kurz darauf auch der rechte. Streif mit dem eigentlichen Primitivstreifen, welcher nun in der geschilderten Weise ungefähr in der Richtung des linken Streifens nach hinten rechts verläuft.

\section{Ergebniss der Untersuchung.}

Aus der Betrachtung des Flächenbildes, der Serie und der Rekonstruction ergiebt sich 1) eine Verdoppelung im Bereich der EmbryonalanIage, welche in der Deutung des Thatsächlichen keine Schwierigkeiten bietet, 2) aber e in e eigenth ïmliche dem Primitivstreifen angehörige, zum Theil mit einer Rinne versehene Bildung, deren Verständniss nicht so leicht ist.

Die Verdoppelung reicht ziemlich weit nach hinten und betrifft auch den vorderen Theil der Primitivrinne. Der Primitivstreif trägt ja nur in seinem hinteren Abschnitt eine e inf a che typische Primitivrinne, welche am vorderen Ende sich gabelnd in $2 \mathrm{Schenkel}$ a usläuft, von denen jeder einem Embryo zukommt. Es handelt sich hier also in der That um eine hinten einfache, vorn doppelte Primitivrinne, deren Existenz zwar von Gerlach vermuthet, welche aber noch von keinem Forscher mit Sicherheit beobachtet worden ist. Mithin ist die Doppelbildung als ein Anadidy mus mit z i e mlich weit nach hinten reichender Spaltung aufu- 
fassen, dessen Componenten aber sehr wenig divergiren. Während die lateralen Hälften beider Embryonen sich in der gewöhnlichen Weise ausbilden konnten, war die reguläre Entwicklung der medialen Hälften wegen der grossen Nähe beider Komponenten unmöglich. Daher ist es zur Bildung eines gemeinsamen Kopfes, eines gemeinsamen breiten Vorderdarmes, einer gemeinsamen weit klaffenden Merlullarrinne gekommen. Die Chorda als der Achsenfaden, um welchen sich alle übrigen Organe des Embryo anordnen, ist am vollständigsten verdoppelt.

Neben dieser typischen Doppelbildung sind gleichsam als eine Komplikation jene strangförmigen Ausstrahlungen des Primitivstreifens vorhanden, über deren Bedentung wir uns jetzt $z$ äussern haben. Da diese Anhangsgebilde in ihrer Form sowohl wie in ihrer histologischen Zusanmensetzung einige Aehnlichkeit mit Primitivstreifen haben, - zumal die am vorderen Ende des rechten Streifens vorhandene Rinne darauf hinweist - so könnte man dieselben als 2 rudimentäre Embryonalanlagen auffassen und somit das ganze Monstrum als eine Vierfachbildung ansprechen. Indessen spricht gegen diese Auffassung die Ungleichheit der 4 Componenten hinsichtlich ihrer Entwicklungsstufe, während doch sonst bei Mehrfachbildungen die grosse Aehnlichkeit der Komponenten immer auffiillt and von den Autoren anch stets betont worden ist. Ferner ist es nicht gut denkbar, wie am hinteren Ende einer Vierfachbildung eine einfache Primitivrinne folgen kann; endlich ist besonders der linke Streifen mit einem Primitivstreifen nicht entfernt zu vergleichen, und auch der rechte ist nur an seinem vorderen Ende einem solchen sehr ähnlich.

Man wird daher dies Gebilde zur Gruppe der bei Reptilien stets, aber auch bei Vögeln nicht ganz selten beobachteten sichelartigen Bildungen rechnen müssen. Sicheln sind bei Vögeln nur in sehr frühen Entwicklungsstadien (zur Zeit der ersten Anlage des Primitivstreifens) und nur in sehr geringer Ausdehnung beobachtet worden. Aber man findet doch ab und zu grössere Sicheln auch in späteren Stadien; so besitze ich eine Keimscheibe mit vollkommen ausgebildetem Primitivstreifen, an dessen hinterem Ende sich eine grosse quergestellte Sichel mit deutlicher Rinne findet. Die ausserordentliche Grösse der Sichel lässt sich in diesem Falle vielleicht daraus erklären, dass 
anch binten das Material zur Anlage zweier Embryonen vorhanden war, welches, da es sich medianwärts nicht ausbreiten konnte, um so stärker lateralwärts wucherte. Ueber den Crsprung und die Bedeutung jener kurzen Rinne am vorderen Ende des rechten Stranges vermag ich Bestimmteres nicht zu sagen.

Man kann nun noch die Frage aufwerfen, ob es sich nur um eine zufaillige Komplikation der typischen Doppelbildung mit der extremen Sichelform handelt, oder ob beide in ursächlichem Zusammenhang stehen. Wie aus den zuletzt gemachten Bemerkungen hervorgeht, neige ich der letzteren Auffassung zu, und nehme an, dass nur, weil das Material für eine Doppelbildnng vorhanden ist, eine so starke Wucherung des Mesoderms stattfinden konnte.

Zum Sehluss will ich kur\% die Frage der Entstehung sw e is e dieses Doppelmonstrums beriuhren. An Theorieen fehlt es gerade für die Anadidymi des Hühnehens nicht, aber keine dieser Theorieen ist genügend durch Beobachtungen und Argumente gestiitzt, wie ich in der Einleitung darzuthun nich bemitht habe. Es handelt sich hier um die Beantwortung dreier Fragen: 1) \% 1 weleher Zeit, 2) ans welchen Griinden, 3) in welcher Form ist diese Doppelbildung angelegt worden.

Keine dieser Fragen lässt sich zur Zeit mit Sicherheit beantworten. Was den Z e itpunkt betrifft, so hat man (besonders $R \circ u x$ ) angenommen, dass Doppelbildungen mit Duplicität der Achsenorgane schon vor oder bei der Entstehung der ersten Furche angelegt sein müssen. Dass eine so frühe Anlage von Doppelbildungen möglich, vielleicht sogar wahrscheinlich ist, will ich nicht bestreiten, aber ich kenne keinen zwingenden Grund für die Annahme, dass alle Doppelbildungen so früh angelegt werden. Will man a priori als gewiss ansehen, dass alle Doppelbildungen v or der ersten Furche entstanden sind, dann hört die Untersuchung der späteren Formen auf, Object der Forschung zu sein. Die aprioristische Annahme ist jedoch nicht zwingend, vielmehr müssen wir einstweilen noch im Auge behalten, ob nicht Doppelbildungen auch später noch angelegt werden kümnen. Demgemäss muss an dem spätest möglichen Termin so lange festgehalten werden, als nicht thatsächliche Beobachtungen zur Annahme eines früheren Zeitpunktes zwingen. Das Material für die Embryonalanlage 
ist nun bei Wirbelthieren kurz vor dem Sichtbarwerden der ersten Spuren des Embryo auf einen sehr kleinen Bezirk entsprechend der vorderen Urmundlippe beschränkt, während die Hauptmasse der Furchungskngeln zur Bildung des Dottersacks und der Hüllen des Embryo dient. Es ist also sehr wohl denkbar, dass kurz vor oder während der Gastrulation geringe Störungen in der Gegend der vorderen Urmundlippe noch zu einer weitgehenden Verdoppelung führen können.

Als Ursachen der Entstehung von Doppelbildungen kanm man sowohl ausserhalb des Keins als auch in diesem selbst liegende Kräfte annchmen. Aeussere Momente kann ich solange nicht anerkennen, als ihre Wirksamkeit nicht experimentell nachgewiesen ist. Bisher sind aber alle Bemintungen, Doppelmonstra experimentell lierzustellen, missgliiekt. Sowohl mechanisehe Spaltungen und Respirationsbeschrinkungen als auch chemische Einflüsse (Chloroform etc. - Hertwig) haben zn keinen sicheren Resultaten geführt. Es bleibt daher nur übrig, im Keim gelegene L rs a chen anzunehmen, wofiir jil aluch die Beobachtungen über Vererbung von Doppelbildungen sprechen. In welchem Zeitpunkte jene im Keim gelegrenen besonileren Kräitte iluf den gewöhnlichen Gang der Entwicklung störend einwirken, ist damit nicht gesagt; denn auch die Vererbung doppelter Finger hat man durch Generationen bindurch beobachtet ( $P$ anum), und doch wird deshalb niemand amehmen, dass Fingerverdoppelungen schon bei der Entstehung der ersten Furchen angelegt werden.

Dic Form, in welcher Doppelbildungen auftreten, lässt sich erst vom Erscheinen des Primitivstreitens an verfolgen, da vorher noch keine Spur des künftigen Embryo sichtbar ist. Giewöhnlich erscheinen beim Hühnchen 2 gesonderte Primitivstreifen, welche mit ihren Kopfenden konvergiren und sich späterhin, soweit es der Raum erlaubt, wa 2 getrennten Embryonen ausbilden. In diesem Falle aber ist eine vorn doppelte, hinten einfache Primitivrinne vorhanden. Da nun nach den Untersuchungen Duval's der Kopftheil des Primitivstreifens zuerst entsteht, später erst der hintere Theil, so müssen anfangs 2 getrennte, aber sehr nahe bei einander gelegene Primitivstreifen vorhanden gewesen sein, welche bei ihrem Wachsthum nach hinten zu einem verschmolzen. Auch vorn ist wegen der starken Wucherung des 
Mesoderms jet\%t nur ein Primitivstreif vorhanden, dessen doppelte Rinne aber mit Sicherheit anf die Entstehung aus zweien hinreutet. Von jerlem Primitivstreifen aus ist dann je eine Chorda und je eine Medullarrinne entstanden; wegen der geringen Divergenz beider Chorden wurde die Medullarrinne bei der weiteren Ausbildung einfach, nul am Kopf blieb sie doppelt; auch der Vorderdarm hat sich aus dem gleichen Grunde einfach angelegt. Aus dem Gesagten geht hervor, dass ich mich fuir eine Spaltung entscheide, welche aber nicht durch äussere Einflusse bewirkt wird, sondern aus inneren Griunden ertolgt.

\section{Nachschrift.}

Diese Arbeit ist am 22. Mär\% 1892 fortig gestellt, Hern l'rof. H. Virchow vorgelegt und von ilhm der Fakultait zur Annahme als Dissertation empfohlen worden. Die Veröftentlichung verö̈gerte sich aus äusseren Gründen Monate lang. So erklärt es sich, dass ich bei der Besprechung der ïber die Entstehung der Mehrfachbildungen aufgestellten Theorieen die inswischen erschienene Studie O. Hertwig's über "Urmund und spina bifida" nicht erwähnt habe.

Es kann hier nicht meine Aufgabe sein, genauer auf die Ergebnisse der Hertwig'sehen Arbeit einzugehen, nur 2 Punkte, rlie hauptsächlich für meinen Fall in Betracht kommen, will ich hervorheben; der erste betrifft die Entstehung der Embryonalanlage, der zweite die Genese der Mehrfachbildungen.

Ich labe in Vorhergehenden in Uebereinstimmung mit Hertwig angenommen, dass in dotterreichen Eiern die Embryonalanlage aus der nächsten Umgebung des Urmundes herrorgeht, weiche aber darin von ihm ab, dass ich die Embryonalanlage hauptsächlich aus der vorderen Urmundlippe entstehen lasse, während Hertwig vor allem die seitlichen Begrenzungen des Urmunds dafür verantwortlich macht. Der Urmund, sagt Hertwig, ändert im Lauf der Entwicklung beständig seine Form, Lage und Ausdelnnung. Im Kopfbereich der Embryonalanlage entstanden, schliesst er sich bald nach, seiner ersten Anlage durch Verwachsung seiner seitlichen Ränder vom vorderen Ende an, während er sich nach hinten vergrössert und 
eine Zeit lang offen erhält. Längs der so entstandenen Verschlussnaht der seitlichen Urmundränder differenziren sich die Primitivorgane des Embryo.

Diese Auffassung erscheint in hohem Maasse gewinnend und überzeugend; es liegt jedoch in dem von mir untersuchten Fall kein Anhaltspunkt vor, der mich berechtigte, in dieser Frage eine Entscheidung zu treffen.

Ueber die Ursachen der Entstehung der I ehrfachbildungen kann auch Hertwig keine bestimmten Angaben machen; er weist wohl anf die Bedentung. der Leberfruchtung. geschädigter Eier hin, vermag aber einen einfachen Zusammenhang zwischen Ueberfruchtung und Mehrfachbildumg nicht $z u$ finden.

Was nun den Zeitpunkt der Entstehung der Mehrfachhildungen betrifft, so muss man es als einen glicklichen Gedanken Hertwig's bezeichnen, dass er gerade den Beginn der Crastrulation als den Zeitpunkt des Sichtbarwerdens der Mehrfachbildungen annimmt. Eine mehrfache Gastrulation muss auch in dem von mir beobachteten Fall stattgefunden haben, da zwei Chordae vorhanden sind; auch die vorn bestehende Verdoppelung der Primitivinne weist noch mit Sicherbeit darauf hin. Man muss, wie ich oben ausgeführt habe, an diesem Termin als den spätest möglichen so lange festhalten, bis thatsäcliche Beobachtungen zur Annahme cines frïheren zwingen.

Ueber die Art und Weise, in welcher der von mir beschriebene Anadidymus entstanden sein mag, glaube ich anf Grund der Ergebnisse der Hertwig'schen Arbeit nun folgende Angaben machen za können:

Nach vollendeter Furchung hat aus nicht bekannter Ursache am Keimscheibenrand statt einer einfachen eine doppelte Gastrulaeinstülpung stattgefunden, welche zur Entstehung zweier Primitivrinnen führte. Beide Gastrulaeinstïlpungen lagen sehr nahe bei einander; daher ist es weiter hinten in Folge des "conjunctiven Wacbsthums" (nach Vesbrauch der sehr kurzen inneren Zwischenstrecke) zur Entstehung einer einfachen Primitivrinne gekommen. Die doppelte Gastrulaeinstilpung hat zur Entwicklung einer sehr weit verdoppelten Chorda getührt, nm welche sich die ibbrigen Primitivorgane in der oben geschilderten Weise angelegt habel. 
Am Schluss dieser Arbeit ist es mir eine angenehme Pflicht, Herrn Prof. Dr. H. Virchow für die überans liebenswürdige Unterstützung bei der Anfertigung dieser Arbeit hiermit meinen verbindlichsten Dank ausuasprechen.

\section{Erklärung der Fignren auf Tafel VIII.}

Fign. 1. Obertlichenansicht der Doppelbildung rom Rücken her bei 20 facher Vergrösserung im durchfallenden Licht. Die nebengezcichneten Striche zeigen die Stelle der abgebildeten Schnitte (Fig. 2-11) an.

Fig. 2. Selhitt durch den Kopt der Doppelbildung in 0,18 nm Entfernung rom Kopfende, trifft den Kopfdarm.

Fig. 3. Schnitt durch den Kopf in $0,28 \mathrm{~mm}$ Entfernung vom Koptende.

Fig. 4. Schnitt durch den Kopttheil in 0,38 $\mathrm{mn}$ Entfernung vom Kopfende.

Fig. 5. Schnitt durch den Koptheil in 0,5 mm Entfernung vom Kopfente, da wo die Vereinigung beider Medallarinnen in eine breite Rinne beginnt.

Fig. 6. Schnitt in 0,6 $\mathrm{mm}$ Entfernung vom Koptende in der Gegend der breiten gemeinsamen Medullarrinne.

Fig. 7. Schnitt in $0,72 \mathrm{~mm}$ Fntfernung vom Kopfende.

Fig. 8. Schnitt in 0,9 min Entfernung vom Koplende vor den Urwirbeln.

Fig. 9. Schnitt in 1,2 mm Entfernung vom Kopfende in der Urwirbelgegend.

Fig. 10. Schnitt in 1,08 $\mathrm{mm}$ Entfernung vom Kopfende dicht vor der Spaltung der Chorda.

Fig. 11. Schnitt in 1,68 mm Entfernung vom Kopfende trifft die Rinne in dem nach links vom Primitivstreifen gelegenen Strang.

Fig. 12. Rekonstruction des hinteren Theils der Doppelbildung; die rothen Linien bezeichnen die Grenzen der Chorden. 